\title{
Filter Bank Precoding for FIR Equalization in High-Rate MIMO Communications
}

\author{
A. Vijaya Krishna and K. V. S. Hari, Senior Member, IEEE
}

\begin{abstract}
In this paper, the problem of designing finite-impulse-response (FIR) equalizers for multiple-input multiple-output (MIMO) FIR channels is considered. It is shown that an arbitrary MIMO frequency-selective channel can be rendered FIR equalizable by a suitable filter bank (FB) precoding operation that introduces redundancy at the transmitter. The expression for the minimum redundancy required to ensure FIR invertibility is derived. The analysis is extended to the case of MIMO multicarrier modulation. Optimum zero-forcing (ZF) and minimum mean-squared error (MMSE) solutions for the FIR equalizer are derived. Simulation results are provided to demonstrate that the proposed scheme achieves better performance than the block-processing methods while supporting a higher data rate.
\end{abstract}

Index Terms-Filter bank, finite-impulse-response (FIR) equalization, multiple-input multiple-output (MIMO), polynomial matrix, pseudocirculant matrix, Smith form.

\section{INTRODUCTION}

$\mathbf{W}$ ITH the ever-increasing demand for higher data rates, multple-input multiple-output (MIMO) designs are perhaps the most viable options for future wireless communication systems [1]. Various channel impairments like multipath and dispersion, resulting in intersymbol interference (ISI), make signal processing for MIMO communications a challenging task. Techniques like MIMO-orthogonal frequency-division multiplexing (MIMO-OFDM) and space-time (ST) precoding have been developed to deal with MIMO frequency-selective channels [2], [3]. In this paper, we provide a filter bank (FB) framework for MIMO communications.

In the single-input single-output (SISO) case, the FB precoding approach to multicarrier modulation is quite well developed [4], [5]. FB transceivers can be designed to provide a much higher data rate (number of symbols per channel use) than the block processing methods like OFDM that require redundancy of the order of channel length [4], [6]. In addition, the FB framework contains the block processing methods as special cases, thus providing a larger context for studying the tradeoffs involved in system design.

Among the FB approaches to MIMO communications, the knowledge of the $M \times N$ channel $H(z)$ is utilized in [7] to design a pre-equalizer, i.e., a polynomial matrix $T(z)$ such that $H(z) T(z)=I$, at the transmitter. However, this method requires $M \leq N$, and assumes that the channel coefficient

Manuscript received December 1, 2004; revised May 20, 2005. The associate editor coordinating the review of this manuscript and approving it for publication was Dr. Markus Rupp.

The authors are with the Department of Electrical Communication Engineering, Indian Institute of Science, Bangalore 560 012, India (e-mail: vkrishna@ece.iisc.ernet.in; hari@ece.iisc.ernet.in).

Digital Object Identifier 10.1109/TSP.2006.871971 matrices are orthogonal. In [8], a blind finite-impulse-response (FIR) equalizer is designed using polynomial matrix techniques. Pohl et al.[9] uses the Kronecker form of matrix pencils to design a zero-forcing (ZF) equalizer. These methods, which perform FIR equalization at the receiver, in general require $M>N$ and the channel matrix to be irreducible. In [10], an iterative procedure for the joint design of precoder and equalizer is developed with the assumption that the channel is communicable. In [11], the concept of biorthogonal partners is used to design an FIR fractionally spaced equalizer.

In this paper, we consider the problem of designing a FB precoding framework that achieves FIR equalization of an arbitrary MIMO FIR channel without imposing any constraints on the nature of the channel. The channel can be of any dimension, and it can even be singular. At the transmitter, the availability of channel knowledge is utilized to design an FIR precoder in such a way as to make the precoded channel FIR invertible. We derive the minimum redundancy required to accomplish FIR invertibility. In practical scenarios wherein the assumption of random channel coefficients holds, it will be seen that full rate can be achieved for rectangular channels in the FB precoding framework, i.e., no redundancy is required. However, when the channel is square, a redundancy of one symbol per channel use is required. In addition, it will be seen that the assumption of channel knowledge at the transmitter can be dispensed with in practical scenarios.

A redundancy of one symbol per channel use for square channels implies considerable rate loss when the channel dimensions are small. This motivates us to extend the FB precoding framework to the case of MIMO multicarrier modulation. Due to the blocking operation inherent in multicarrier modulation, the effective channel becomes a block pseudocirculant matrix [12]. By investigating the properties of the Smith form of block pseudocirculant matrices, we derive the expression for minimum redundancy required to enable FIR equalization at the receiver. It will be seen that the MIMO multicarrier approach retains the full rate advantage for rectangular channels, while increasing the achievable data rate in case of square channels. In addition, the MIMO multicarrier approach provides a better framework than the basic FB approach for trading off rate for performance. Compared with the ST methods, which require redundancy of the order of channel length [2], the MIMO multicarrier framework provides comparable performance while supporting a much higher data rate.

The equalizer for the precoded channel is in the form of a FIR left inverse, and hence is not unique. This non-uniqueness can be utilized to design equalizers based on different criteria. We show that the design freedom available at the precoder can be 
used to aid in the design of noise-minimizing ZF equalizer. We also derive the minimum mean-squared error (MMSE) equalizer for the precoded channel.

\section{Precoding Framework}

Consider the MIMO signal model given by

$$
y(n)=\sum_{k=0}^{L_{H}-1} h(k) x(n-k)+v(n)
$$

where $x(n)$ is the $N$-length input vector, $y(n)$ is the $M$-length received signal vector, $h(k), k=0,1, \ldots, L_{H}-1$ is the timedomain representation of the $M \times N$ frequency-selective MIMO channel, and $v(n)$ is the $M$-length noise vector. In the absence of noise, we can write

$$
Y(z)=H(z) X(z)
$$

in the $z$ domain. Now, an FIR equalizer (left inverse) for $H(z)$ exists if and only if $M \geq N$ and $H(z)$ is irreducible. Therefore, given an arbitrary $H(z)$, we wish to find an $N \times K(K \leq N)$ precoder matrix $G(z)$, so that the $M \times K$ composite channel matrix $C(z)=H(z) G(z)$ has an FIR left inverse. $N-K$ is the redundancy introduced by the precoder. If the channel matrix $H(z)$ is already FIR invertible, then we can chose $G(z)=$ $I(N=K)$, so that no redundancy is introduced. We address the question, What is the maximum $K$ (minimum redundancy $N-K$ ) for which $C(z)$ is irreducible? Before answering this question, we will look at some properties of the Smith form of a polynomial matrix.

\section{A. Smith Form}

Given a $p \times q$ polynomial matrix $A(z)$ with normal $\operatorname{rank} r(r=$ $\left.\max _{z} \operatorname{rank}[H(z)]\right), r \leq \min (p, q)$, we can write [12]

$$
A(z)=E(z) B(z) F(z)
$$

where $E(z)$ and $F(z)$ are unimodular matrices (square matrices with constant determinants) of sizes $p \times p$ and $q \times q$, respectively. $B(z)$ is a $p \times q$ matrix of the form

$$
B(z)=\operatorname{diag}\left(\psi_{1}(z), \psi_{2}(z), \ldots, \psi_{r}(z), 0, \ldots 0\right)
$$

such that $\psi_{i}(z) \mid \psi_{i+1}(z)$ (i.e., $\psi_{i}(z)$ divides $\psi_{i+1}(z)$ ). The monic polynomials $\psi_{i}(z)$ are unique and are called the invariant polynomials of $A(z)$. We say that $A(z)$ is equivalent to $B(z)$ and write $A(z) \sim B(z)$.

Since $E(z)$ and $F(z)$ are unimodular, it follows from (3) that $\operatorname{rank}[A(z)]=\operatorname{rank}[B(z)] \forall z$. Therefore, if the minimum rank of $A(z)$ over all values of $z$ is $r_{m}$, it can be shown that $\psi_{1}(z)=$ $\psi_{2}(z)=\cdots=\psi_{r_{m}}(z)=1$. If an $n \times k(n>k)$ matrix $P(z)$ is irreducible (polynomially invertible), then its Smith form is

$$
P(z) \sim\left[\begin{array}{c}
I_{k \times k} \\
0_{(n-k) \times k}
\end{array}\right]
$$

The following result is stated in [13].

Lemma 1: Let $P_{i}(z), i=1,2$, have invariant polynomials $\left\{\psi_{i 1}(z), \psi_{i 2}(z), \ldots ., \psi_{i r_{i}}(z)\right\}, i=1,2$, where $r_{i}$ is the normal rank of $P_{i}(z)$. Let $P(z)=P_{1}(z) P_{2}(z)$ have normal rank $r$, with invariant polynomials $\left\{\psi_{1}(z), ., \psi_{r}(z)\right\}$. Then, for $1 \leq k \leq r$ and $i=1,2, \psi_{i k}(z) \mid \psi_{k}(z)$.

The proof follows directly from the Binet-Cauchy formula [13].

\section{B. Minimum Redundancy}

Theorem 1: Let $r_{m}$ be the minimum rank of the given $M \times$ $N$ FIR channel $H(z)$. An $N \times K$ precoder $G(z)$ that renders the composite channel matrix $C(z)=H(z) G(z)$ polynomially invertible exists if and only if $K \leq r_{m}$.

Proof: We will first prove the only if part.

Let $\left\{c_{i}(z)\right\},\left\{h_{i}(z)\right\}$ and $\left\{g_{i}(z)\right\}$ denote the invariant polynomials of $C(z), H(z)$ and $G(z)$ respectively. Suppose $C(z)$ is irreducible. Then

$$
C(z) \sim\left[\begin{array}{c}
I_{K \times K} \\
0_{(M-K) \times K}
\end{array}\right] .
$$

We can always choose

$$
G(z) \sim\left[\begin{array}{c}
I_{K \times K} \\
0_{(N-K) \times K}
\end{array}\right]
$$

so that $g_{i}(z) \mid c_{i}(z), 1 \leq i \leq K$.

Since the minimum rank of $H(z)$ is $r_{m}$, we have

$$
h_{1}(z)=\ldots=h_{r_{m}}(z)=1
$$

and

$$
h_{r_{m}+1}(z), \ldots, h_{r}(z) \neq 1
$$

where $r$ is the normal rank of $H(z)$. Hence, $h_{i}(z) \mid c_{i}(z)$ for $1 \leq$ $i \leq K$ only if $K \leq r_{m}$. Therefore, $C(z)$ is irreducible only if $K \leq r_{m}$.

Now we will prove the if part.

Let $H(z)=U(z) D(z) V(z)$ be the Smith form decomposition of the channel matrix. Choose

$$
G(z)=V^{-1}(z)\left[\begin{array}{c}
I_{K \times K} \\
0_{(N-K) \times K}
\end{array}\right] W(z)
$$

where $W(z)$ is an arbitrary $K \times K$ unimodular matrix. Then, we have

$$
C(z)=H(z) G(z)=U(z) D(z)\left[\begin{array}{c}
I_{K \times K} \\
0_{(N-K) \times K}
\end{array}\right] W(z) .
$$

Now, since $r_{m}$ is the minimum rank of $H(z)$, the submatrix formed by the first $r_{m}$ columns of $D(z)$ is

$$
\left[\begin{array}{c}
I_{r_{m} \times r_{m}} \\
0_{\left(M-r_{m}\right) \times r_{m}}
\end{array}\right] \text {. }
$$

If $K \leq r_{m}$, we have

$$
D^{\prime}(z)=D(z)\left[\begin{array}{c}
I_{K \times K} \\
0_{(N-K) \times K}
\end{array}\right]=\left[\begin{array}{c}
I_{K \times K} \\
0_{(M-K) \times K}
\end{array}\right] .
$$

Therefore, $C(z) \sim\left[\begin{array}{c}I_{K \times K} \\ 0_{(M-K) \times K}\end{array}\right]$. Therefore, $C(z)$ is irreducible. 
This result can be seen as a generalization to the MIMO case of the minimum redundancy results obtained for the multicarrier modulation case [4], [6].

\section{Minimum Rank and Channel Zeros}

Given an $M \times N$ channel $H(z)$ with normal rank $r$, let $|H(z)|$ denote the g.c.d (greatest common divisor) of the minors of order $r$ of $H(z)$ (if $H(z)$ is square and has full normal rank, then $|H(z)|$ is its determinant). Expanding $|H(z)|$ in terms of its factors, we have

$|H(z)|=\left(1-\alpha_{0} z^{-1}\right)^{\gamma_{0}}\left(1-\alpha_{1} z^{-1}\right)^{\gamma_{1}} \ldots\left(1-\alpha_{s-1} z^{-1}\right)^{\gamma_{s-1}}$.

The rank of $H(z)$ drops below the normal rank $r$ only at the channel zeros $\alpha_{i}, i=0,1, \ldots, s-1$. We now relate the minimum rank of $H(z)$ to the maximum among the multiplicities of the channel zeros.

Corollary 1: Let $r$ and $r_{m}$ be the normal rank and the minimum rank of $H(z)$ respectively. Let $|H(z)|$ be as in (6). Then, we have $r_{m} \geq r-\max _{i}\left\{\gamma_{i}\right\}$.

Proof: Let $\psi_{i}(z), i=1,2, \ldots r$, be the invariant polynomials of $H(z)$. Then, from the definition of the Smith form, we have

$$
|H(z)|=\prod_{i=1}^{r} \psi_{i}(z)
$$

Now, since $\psi_{i}(z) \mid \psi_{i+1}(z)$, it follows that if some $\beta(z)$ is a factor of $\psi_{r-j}(z)$, then its multiplicity as a factor of $|H(z)|$ is at least $j+1$. Thus, any factor $\left(1-\alpha_{j} z^{-1}\right)^{\gamma_{j}}$ of $|H(z)|$ cannot be a factor of $\psi_{i}(z)$ for $i \leq r-\gamma_{j}$. Therefore, rank of $H\left(\alpha_{j}\right)$ cannot be less than $r-\gamma_{j}$. Thus, we have the lower bound for the minimum rank as $r_{m} \geq r-\max \left\{\gamma_{i}\right\}$.

A random nonsquare polynomial matrix is irreducible with probability 1 [14]. Therefore, given an arbitrary $M \times N$ channel $H(z)$ with $M \neq N$, FB precoding utilizes all the degrees of freedom i.e., the throughput is $\min (M, N)$ symbols per channel use. If $M<N$, then the channel can be completely preequalized i.e., $H(z) G(z)=I$. If $M>N$, FIR equalization can be accomplished without precoding. Compared with ST processing methods [2], [3], the FB precoding scheme provides better throughput at a much lesser complexity. When $M=N$, we need a redundancy of 1, i.e., $K=N-1$, so that the precoded channel becomes a tall matrix.

From the proof of Theorem 1, it is observed that the freedom to choose the right unimodular matrix $W(z)$ is available at the precoder. One way of utilizing this freedom, when channel knowledge is available at the transmitter, is given in Section IV.
When the transmitter has no channel knowledge, this freedom can be used for channel independent techniques like constellation precoding [15].

\section{MIMO MULTICARRIER FRAMEWORK}

Consider the MIMO signal model given by (1). Suppose the $M \times 1$ output vectors $y(n)$ are blocked to form the $M L \times 1$ vector $\mathcal{Y}(n)=\left[y^{T}(n) y^{T}(n-1) \ldots y^{T}(n-L+1)\right]^{T}$, where $L$ is the block-length (it corresponds to the number of tones in case of MIMO-OFDM). Let $H_{k}(z), k=0,1, \ldots, L-1$ be the polyphase components of $H(z)$ of order $L$, given by

$$
H_{k}(z)=\sum_{n} h(L n+k) z^{-n}
$$

The polyphase components of $Y(z)$ and $X(z)$ are defined similarly. Now, consider the convolution

$$
y(n)=\sum_{k} h(k) x(n-k) .
$$

We can express the odd and even parts of $y(n)$ separately as

$$
\begin{aligned}
y(2 n)= & \sum_{k} h(2 k) x(2 n-2 k) \\
& +\sum_{k} h(2 k+1) x(2 n-2 k-1) \\
y(2 n+1)= & \sum_{k} h(2 k+1) x(2 n-2 k) \\
& +\sum_{k} h(2 k) x(2 n-2 k+1)
\end{aligned} .
$$

Applying $z$ transform and using the definition of polyphase components, we can write

$$
\begin{aligned}
& Y_{0}(z)=H_{0}(z) X_{0}(z)+z^{-1} H_{1}(z) X_{1}(z) \\
& Y_{1}(z)=H_{1}(z) X_{0}(z)+H_{0}(z) X_{1}(z)
\end{aligned} .
$$

In general, for a polyphase decomposition of order $M$, we can write

$$
Y_{k}(z)=\sum_{i=0}^{k} H_{k-i}(z) X_{i}(z)+z^{-1} \sum_{i=k+1}^{M-1} H_{M-i}(z) X_{i}(z) .
$$

The resulting system model is given by (8), shown at the bottom of the page.

The relation given in (8) can be denoted concisely as

$$
\mathcal{Y}(z)=\mathcal{H}_{L}(z) \mathcal{X}(z)
$$

The $M L \times N L$ matrix $\mathcal{H}_{L}(z)$ is called a block pseudocirculant matrix. Pseudocirculant matrices (which result when $H(z)$

$$
\left[\begin{array}{c}
Y_{0}(z) \\
Y_{1}(z) \\
\vdots \\
Y_{L-1}(z)
\end{array}\right]=\left[\begin{array}{ccccc}
H_{0}(z) & z^{-1} H_{L-1}(z) & z^{-1} H_{L-2}(z) & \cdots & z^{-1} H_{1}(z) \\
H_{1}(z) & H_{0}(z) & z^{-1} H_{L-1}(z) & \cdots & z^{-1} H_{2}(z) \\
\vdots & & & & \vdots \\
H_{L-1}(z) & H_{L-2}(z) & H_{L-3}(z) & \cdots & H_{0}(z)
\end{array}\right]\left[\begin{array}{c}
X_{0}(z) \\
X_{1}(z) \\
\vdots \\
X_{L-1}(z)
\end{array}\right]
$$


is a scalar polynomial) are studied in detail in [16]. The following result, which is an extension of the diagonalization result of pseudocirculants to the block pseudocirculant case, was proved in [14]:

$$
\mathcal{H}_{L}\left(z^{L}\right)=P_{l}[W]_{L}^{-1} P_{l} V(z) P_{r}[W]_{L} P_{r}
$$

with

$$
[W]_{L}=\operatorname{diag}\left(W_{L} \Lambda_{L}(z), \ldots, W_{L} \Lambda_{L}(z)\right)
$$

where

$$
\Lambda_{L}(z)=\operatorname{diag}\left(1, z^{-1}, z^{-2}, \ldots, z^{-L+1}\right)
$$

and $W_{L}$ is the $L \times L$ DFT matrix. $P_{l}$ and $P_{r}$ are the row and column block permutation matrices, and

$$
V(z)=\operatorname{diag}\left(H(z), H\left(z w^{-1}\right), \ldots, H\left(z w^{-L+1}\right)\right)
$$

with $w=e^{-j 2 \pi / L}$.

From (12), we can infer the following [14]:

- since $P_{l}[W]_{L}^{-1} P_{l}$ and $P_{r}[W]_{L} P_{r}$ are irreducible, $\operatorname{rank}\left[\mathcal{H}_{L}\left(z^{L}\right)\right]=\operatorname{rank}[V(z)] \forall z ;$

- $\mathcal{H}_{L}(z)$ is irreducible if and only if $H(z)$ is irreducible.

\section{A. Minimum Redundancy for MIMO Multicarrier Modulation}

From Section II-B, we know that a rate loss of $(N-K) / N$ is incurred for accomplishing FIR invertibility. This rate loss can be considerable when $N$ is small. This motivates us to find the minimum redundancy required for the $M L \times N L$ block pseudocirculant channel matrix $\mathcal{H}_{L}(z)$ in the MIMO multicarrier representation $\mathcal{Y}(z)=\mathcal{H}_{L}(z) \mathcal{X}(z)$.

Lemma 2: Given an $M \times N$ polynomial matrix $H(z)$, if $|H(z)|$ has roots $\left\{\alpha_{0}, \alpha_{1}, \ldots, \alpha_{s-1}\right\}$, then $\left|\mathcal{H}_{L}(z)\right|$ has roots $\left\{\alpha_{0}^{L}, \alpha_{1}^{L}, \ldots, \alpha_{s-1}^{L}\right\}$. This result can be proved in a manner similar to the SISO case [6].

For any polynomial matrix $H(z)$ with normal rank $r$, its rank falls below $r$ only at the roots of $|H(z)|$. We have

$|H(z)|=\left(1-\alpha_{0} z^{-1}\right)^{\gamma_{0}}\left(1-\alpha_{1} z^{-1}\right)^{\gamma_{1}} \ldots\left(1-\alpha_{s-1} z^{-1}\right)^{\gamma_{s-1}}$.

At any root $\alpha_{i}$, the rank of $H(z)$ drops by 1 if the multiplicity of $\alpha_{i}$ is 1 . When the multiplicity of the root is more than 1 , there is possibility of the rank drop being more than 1. Let $\nu\left(\alpha_{k}\right)$ denote the rank drop of $H(z)$ at $z=\alpha_{k}$. From the proof of corollary 1 , it follows that $\nu\left(\alpha_{k}\right) \leq \gamma_{k}$. The minimum rank $r_{m}$ of $H(z)$, which is the minimum value of $\operatorname{rank}[H(z)]$ over all $z$, is given by $r_{m}=r-\max _{k}\left\{\nu\left(\alpha_{k}\right)\right\}$.

Definition: A set of zeros $\left\{\alpha_{k_{1}}, \alpha_{k_{2}}, \ldots, \alpha_{k_{q}}\right\}$ of $|H(z)|$ is called congruous with respect to $L$ if the following hold [6]:

1) $\alpha_{k_{1}}, \alpha_{k_{2}}, \ldots, \alpha_{k_{q}}$ are distinct;

2) $\alpha_{k_{1}}^{L}=\alpha_{k_{2}}^{L}=\ldots=\alpha_{k_{q}}^{L}$.

If two zeros $\alpha_{i}$ and $\alpha_{j}$ of $|H(z)|$ are congruous with respect to $L$, then $\alpha_{i}=\left|\alpha_{j}\right| e^{j 2 \pi l / L}$ where $1 \leq l \leq L-1$ [6]. Congruous zeros have the same magnitude and are phase shifted by an integer multiple of $2 \pi / L$.

Let the zeros of $|H(z)|$ be partitioned into sets of congruous zeros such that the number of congruous sets is minimum, i.e., no two sets can be combined to form a larger congruous set [17].
Each set contains either congruous zeros or a single zero. If, for example, a zero $\alpha_{k}$ has multiplicity 3 and is congruous to $\alpha_{j}$ (and is congruous to no other zero), then we have three congruous sets: $\left\{\alpha_{k}, \alpha_{j}\right\},\left\{\alpha_{k}\right\}$, and $\left\{\alpha_{k}\right\}$. Let there be a total of $q$ congruous sets $B_{1}, B_{2}, \ldots, B_{q}$. Let $n_{j}$ denote the cardinality of $B_{j}$. Each $B_{j}$ is of the form

$$
B_{j}=\left\{\alpha_{j, 1}, \alpha_{j, 2}, \ldots, \alpha_{j, n_{j}}\right\} .
$$

We have $\sum_{j=1}^{q} n_{j}=\sum_{k=0}^{s-1} \gamma_{k}$. For each $B_{j}$, let us define

$$
\mu\left(B_{j}\right)=\sum_{i=1}^{n_{j}} \nu\left(\alpha_{j, i}\right) .
$$

Theorem 2: Given an $M \times N$ polynomial matrix $H(z)$ with normal rank $r$, the minimum rank of the block pseudocirculant matrix $\mathcal{H}_{L}(z)$ is $L r-\max _{j} \mu\left(B_{j}\right)$.

Proof: Let $H(z)$ have the Smith form

$$
\operatorname{diag}\left\{\delta_{1}(z), \delta_{2}(z), \ldots, \delta_{r}(z), 0, \ldots, 0\right\} .
$$

Then it can be verified that $H\left(z^{L}\right)$ has the Smith form

$$
\operatorname{diag}\left\{\delta_{1}\left(z^{L}\right), \delta_{2}\left(z^{L}\right), \ldots, \delta_{r}\left(z^{L}\right), 0, \ldots, 0\right\} .
$$

Since $H(z)$ and $H\left(z^{L}\right)$ have the same number of invariant polynomials, they have the same normal rank. Since the number of nonunity invariant polynomials is also the same, both $H(z)$ and $H\left(z^{L}\right)$ have the same minimum rank.

If $H(z)$ has normal rank $r$, so does $H\left(z w^{-i}\right), i=$ $1,2, \ldots, L-1$. Therefore, it follows from (12) that $V(z)$ has normal rank $L r$. Now, since $\operatorname{rank}\left[\mathcal{H}_{L}\left(z^{L}\right)\right]=\operatorname{rank}[V(z)] \forall z$, $\mathcal{H}_{L}\left(z^{L}\right)$ has normal rank $L r$, the same as that of $V(z)$. In addition, the minimum rank of $\mathcal{H}_{L}\left(z^{L}\right)$ is the same as that of $V(z)$. Therefore, we have the following:

- normal rank of $\mathcal{H}_{L}(z)$ is $L r$;

- minimum rank of $\mathcal{H}_{L}(z)$ is equal to the minimum rank of $V(z)$.

Now, let $\alpha_{k}$ be a root of $|H(z)|$. At $z=\alpha_{k}, \operatorname{rank}[H(z)]$ drops by $\nu\left(\alpha_{k}\right)$. At $z=\alpha_{k}, \operatorname{rank}\left[H\left(z w^{-l}\right)\right]$ also drops if and only if $\alpha_{k}$ is a common zero for both $|H(z)|$ and $\left|H\left(z w^{-l}\right)\right|$. This means that both $\alpha_{k}$ and $\alpha_{k} e^{-j 2 \pi l / L}$ are zeros of $|H(z)|$, i.e., the two zeros are congruous.

Therefore, at any $\alpha_{j, k} \in B_{j}, n_{j}$ matrices among $H(z)$, $H\left(z w^{-1}\right), \ldots, H\left(z w^{-L+1}\right)$ become rank deficient, by $\nu\left(\alpha_{j, 1}\right), \nu\left(\alpha_{j, 2}\right), \ldots, \nu\left(\alpha_{j, n_{j}}\right)$ respectively. Therefore, the rank drop at any $\alpha_{j, k} \in B_{j}$ is $\sum_{i=1}^{n_{j}} \nu\left(\alpha_{j, i}\right)=\mu\left(B_{j}\right)$.

Therefore, we have that the maximum rank drop of $V(z)$ is $\max _{j} \mu\left(B_{j}\right)$. Therefore, the minimum rank of $V(z)$, which is equal to the minimum rank of $\mathcal{H}_{L}(z)$, is $L r-\max _{j} \mu\left(B_{j}\right)$.

We can now extend the minimum redundancy result to the MIMO multicarrier case.

Corollary 2: Given a block-length $L$ and an $M \times N$ FIR channel $H(z)$ with normal rank $r$, an $N L \times K$ precoder $G(z)$ which renders the composite channel matrix $\mathcal{H}_{L}(z) G(z)$ polynomially invertible exists if and only if $K \leq L r-\max _{j} \mu\left(B_{j}\right)$.

Example: Consider a $3 \times 3$ matrix $H(z)$ with the following Smith form:

$$
\operatorname{diag}\left\{1,\left(1-z^{-1}\right),\left(1-z^{-1}\right)\left(1+z^{-1}\right)\left(1-2 z^{-1}\right)\right\} .
$$


We have $|H(z)|=\left(1-z^{-1}\right)^{2}\left(1+z^{-1}\right)\left(1-2 z^{-1}\right)$. The zero at $z=1$ (with multiplicity 2 ) is congruous to the one at $z=-1$ with respect to 2 . From Lemma 2, we have

$$
\left|\mathcal{H}_{2}(z)\right|=\left(1-z^{-1}\right)^{3}\left(1-4 z^{-1}\right)
$$

From Theorem 2, the minimum rank of $\mathcal{H}_{2}(z)$ is 3 , and its Smith form is given by

$$
\operatorname{diag}\left\{1,1,1,\left(1-z^{-1}\right),\left(1-z^{-1}\right),\left(1-z^{-1}\right)\left(1-4 z^{-1}\right)\right\} .
$$

\section{Remarks:}

- A random nonsquare polynomial matrix is almost surely irreducible [14]. Therefore, for an arbitrary square polynomial matrix $H(z)$, we have $\gamma_{k}=1$ and $n_{j}=1 \forall k, j$ with probability 1 , which means, by Theorem 2 , that a redundancy of one symbol per channel use is enough. Therefore, given an $N \times N$ channel matrix, the rate loss to be incurred to enable FIR equalization is $1 / L N$ in the MIMO multicarrier scheme, as opposed to the $1 / N$ factor without multicarrier modulation. For rectangular channels, the MIMO multicarrier scheme retains the fullrate advantage. In addition, the multicarrier approach provides better resolution $(1 / L N)$ for trading off rate for performance than the basic FB approach $(1 / N)$.

- In the ST precoding approach, the precoder and the equalizer can be jointly optimized [2]. In FB precoding, obtaining a closed form solution for the joint optimization of the precoder and the equalizer is difficult, and iterative optimization techniques need to be used [10]. Since the focus in this paper is on the algebraic aspects of FIR invertibility, we have used the simple zero-padding precoder of the form $G(z)=\left[\begin{array}{c}I_{K} \\ 0_{(N L-K) \times K}\end{array}\right]$.

\section{EQUALIZER DESIGN}

The design of the equalizer can be based on different criteria such as low delay, low complexity and noise minimization. The polynomial matrix framework enables one to utilize many of the results in systems theory literature regarding the construction of inverse systems [18]. In this section, we derive the MMSE equalizer and the noise-minimizing ZF equalizer, using the design freedom available at the precoder.

We assume that the receiver has the knowledge of the $M \times K$ precoded channel $C(z)=H(z) G(z)\left(\right.$ or $C(z)=\mathcal{H}_{L}(z) G(z)$, in case of multicarrier modulation). We also assume that the noise $v(n)$ in (1) is zero-mean Gaussian and that the covariance matrix $R_{v v}$ is positive definite. The equalizer design problem is to find a polynomial matrix $F(z)=\sum_{i=0}^{L_{F}-1} f(i) z^{-i}$ such that $F(z) C(z)=I$. This can also be written as $\mathcal{F C}=\mathcal{J}$, where

$$
\begin{aligned}
\mathcal{F} & =\left[\begin{array}{lllll}
f(0) & f(1) & \ldots & f\left(L_{F}-1\right)
\end{array}\right] \\
\mathcal{C} & =\left[\begin{array}{lllll}
c(0) & \ldots & c\left(L_{C}-1\right) & & \\
& \ddots & & \ddots & \\
& & c(0) & \ldots & c\left(L_{C}-1\right)
\end{array}\right]
\end{aligned}
$$

and $\mathcal{J}=[I 0 \cdots 0] . \mathcal{F}$ is $K \times M L_{F}, \mathcal{J}$ is $K \times K\left(L_{c}+L_{F}-1\right)$, and $\mathcal{C}$ is $M L_{F} \times K\left(L_{C}+L_{F}-1\right)$. $\mathcal{C}$ is tall if $L_{F} \geq\left(K\left(L_{C}-\right.\right.$
$1) / M-K) \cdot \mathcal{C}$ is full rank if and only if the following are found [19]:

- $C(z)$ is irreducible and column reduced;

- $\quad L_{F} \geq \max _{1 \leq j \leq M-K} \kappa_{j}^{\perp}-1$, where $\kappa_{j}^{\perp}$ are the Kronecker indexes of the dual space of $\operatorname{span}\{C(z)\}$;

- the degrees of all the columns of $C(z)$ are equal.

The third restriction can be overcome by deleting the null columns of $\mathcal{C}$ as explained in [8]. Therefore, given an irreducible $C(z)$ and with $L_{F}$ chosen large enough, $\mathcal{C}$ is full rank if $C(z)$ is column-reduced. Any polynomial matrix can be brought to column-reduced form by multiplying it to the right by a suitable unimodular matrix [13]. From the proof of Theorem 1, it follows that we have the freedom of choosing a $K \times K$ unimodular factor of $G(z)$. We can choose that unimodular matrix such that $C(z)$ becomes column reduced, resulting in a full rank $\mathcal{C}$. In the following section, superscript $H$ denotes Hermitian transpose.

Theorem 3: If $\mathcal{C}$ is tall and full rank, the equalizer which minimizes the output noise power is given by $\mathcal{F}=\mathcal{J}\left(\mathcal{C}^{H} R_{\mathcal{V} \mathcal{V}}^{-1}\right)^{-1} \mathcal{C}^{H} R_{\mathcal{V} \mathcal{V}}^{-1}$

Proof: The output of the equalizer can be written as

$$
\hat{X}(z)=F(z) C(z) X(z)+F(z) V(z) .
$$

Using (13) and (14), we can write

$$
\hat{x}(n)=\mathcal{F C X}(n)+\mathcal{F} \mathcal{V}(n)
$$

where $\mathcal{X}(n)=\left[x^{T}(n), x^{T}(n-1), \ldots, x^{T}\left(n-\left(L_{C}+L_{F}-2\right)\right]^{T}\right.$ and $\mathcal{V}(n)=\left[v^{T}(n), v^{T}(n-1), \ldots, v^{T}\left(n-L_{F}+1\right)\right]$ are the blocked versions of $x(n)$ and $v(n)$, of dimensions $K\left(L_{C}+L_{F}-\right.$ 1) $\times 1$ and $M L_{F} \times 1$, respectively. The covariance matrix of the noise component of $\hat{x}(n)$ is given by

$$
E\left[\mathcal{F} \mathcal{V} \mathcal{V}^{H} \mathcal{F}^{H}\right]=\mathcal{F} R_{\mathcal{V} \mathcal{V}} \mathcal{F}^{H}
$$

in view of the white noise assumption. The noise-minimizing equalizer is obtained by minimizing $\operatorname{tr}\left[\mathcal{F} R_{\mathcal{V}} \mathcal{F}^{H}\right]$ subject to the constraint $\mathcal{F C}=\mathcal{J}$. Using the Lagrange multiplier method, we get

$$
2 \mathcal{F} R_{\mathcal{V} \mathcal{V}}-\mathcal{L C}^{H}=0
$$

where $\mathcal{L}$ is the $K \times K\left(L_{C}+L_{F}-1\right)$ Lagrange multiplier matrix. From (17), we have

$$
2 \mathcal{F C}=\mathcal{L C}^{H} R_{\mathcal{V} \mathcal{V}}^{-1} \mathcal{C}
$$

Since $\mathcal{F C}=\mathcal{J}$

$$
\mathcal{L}=2 \mathcal{J}\left(\mathcal{C}^{H} R_{\mathcal{V} \mathcal{V}}^{-1} \mathcal{C}\right)^{-1}
$$

From (17) and (19), we have

$$
\mathcal{F}=\mathcal{J}\left(\mathcal{C}^{H} R_{\mathcal{V} \mathcal{V}}^{-1} \mathcal{C}\right)^{-1} \mathcal{C}^{H} R_{\mathcal{V} \mathcal{V}}^{-1}
$$

When the noise $v(n)$ is white, (20) reduces to

$$
\mathcal{F}=\mathcal{J}\left(\mathcal{C}^{H} \mathcal{C}\right)^{-1} \mathcal{C}^{H} \text {. }
$$

Theorem 4: The MMSE solution for the system in (15) is given by $\mathcal{F}=R_{x x} \mathcal{J C}^{H}\left(\mathcal{C} R_{\mathcal{X} \mathcal{X}} \mathcal{C}^{H}+\sigma_{\mathcal{V}}^{2} \mathcal{I}\right)^{-1}$, where $R_{\mathcal{X X}}$ is the covariance matrix of the vectors $\mathcal{X}(n)$. 
Proof: From (15), the desired signal $\hat{x}(n)$ has to be estimated from the observed signal $\mathcal{Y}(n)=\mathcal{C X}(n)+\mathcal{V}(n)$. The Wiener filter in this case is given by

$$
\begin{aligned}
\mathcal{F} & =\left(E\left[x \mathcal{Y}^{H}\right]\right)\left(E\left[\mathcal{Y} \mathcal{Y}^{H}\right]\right)^{-1} \\
& =\left(E\left[x\left(\mathcal{X}^{H} \mathcal{C}^{H}+\mathcal{V}^{H}\right)\right]\right)\left(E\left[(\mathcal{C X}+\mathcal{V})(\mathcal{C X}+\mathcal{V})^{H}\right]\right)^{-1} \\
& =R_{x x} \mathcal{J C}^{H}\left(\mathcal{C}_{\mathcal{X X X}_{\mathcal{X}}} \mathcal{C}^{H}+R_{\mathcal{V} \mathcal{V}}\right)^{-1}
\end{aligned}
$$

where the last step follows because the input and the noise are uncorrelated and $E\left[x \mathcal{X}^{H}\right]=\left[R_{x x} 0 \cdots 0\right]=R_{x x} \mathcal{J}$.

When the noise and the input vectors are white, then

$$
\mathcal{F}=\mathcal{J C}^{H}\left(\mathcal{C C}^{H}+\sigma_{v}^{2} \mathcal{I}\right)^{-1}
$$

In practice, in the case of both ZF and MMSE equalizers, least-square computations are preferred over the explicit computation of inverses.

For both ZF and MMSE equalizers, the equalized signal can be written as $\hat{x}(n)=x(n)+e(n)$, where $e(n)$ is the $K \times 1$ error vector. Both the $\mathrm{ZF}$ and MMSE approaches try to minimize the trace of $R_{e e}$, the error covariance matrix. For the ZF equalizer with white noise, from (16) and (21), we can write

$$
R_{e e, \mathrm{ZF}}=\sigma_{v}^{2} \mathcal{F F}^{H}=\sigma_{v}^{2} \mathcal{J}\left(\mathcal{C C}^{H}\right)^{-1} \mathcal{J}^{H} .
$$

Similarly, for the MMSE equalizer, we can write

$$
\begin{aligned}
R_{e e, \mathrm{MMSE}} & =E\left[x x^{H}\right]-\left(E\left[x \mathcal{Y}^{H}\right]\right)\left(E\left[\mathcal{Y} \mathcal{Y}^{H}\right]\right)^{-1}\left(E\left[\mathcal{Y} x^{H}\right]\right) \\
& =I-\mathcal{J C}^{H}\left(\mathcal{C C}^{H}+\sigma_{v}^{2} \mathcal{I}\right)^{-1} \mathcal{C J}^{H}
\end{aligned}
$$

We define $T_{\mathrm{ZF}}\left(\sigma_{v}^{2}\right)=\operatorname{tr}\left(R_{e e, \mathrm{ZF}}\right) / K$ and $T_{\mathrm{MMSE}}\left(\sigma_{v}^{2}\right)=$ $\operatorname{tr}\left(R_{e e, \mathrm{MMSE}}\right) / K$. $T_{\mathrm{ZF}}$ and $T_{\mathrm{MMSE}}$ provide figures-of-merit for the performance of the respective equalizers. The lower the value of $T_{\mathrm{ZF}}$ or $T_{\mathrm{MMSE}}$, the better the performance.

\section{Simulation Results}

Simulations were carried out with $4 \times 4$ channel coefficient matrices $H_{i}$ with independent Gaussian distributed complex coefficients (Rayleigh fading). The power delay profile used was [20]

$$
\sigma_{l}^{2}=\frac{1-e^{-1}}{1-e^{-L_{H}}} e^{-l} \quad l=0,1, \ldots, L_{H}-1 .
$$

The channel order $L_{H}$ was chosen to be 5 . The noise generated was spatially and temporally white. The results were averaged over 100 random channels. The input constellation used in the simulations is BPSK, unless otherwise mentioned.

Fig. 1 shows $T_{\mathrm{ZF}}\left(\sigma_{v}^{2}\right)$ and $T_{\mathrm{MMSE}}\left(\sigma_{v}^{2}\right)$ for different values of redundancy and SNR, with the block-length $L=4$. It can be seen that, for the same signal-to-noise ratio (SNR), $T_{\mathrm{MMSE}}$ is less than $T_{\mathrm{ZF}}$, confirming that the MMSE equalizer performs better than the ZF equalizer, even though the gap is closer at high SNR. In addition, $T_{\mathrm{ZF}}$ and $T_{\mathrm{MMSE}}$ decrease with increasing redundancy, indicating an improvement in performance with decreasing data rate.

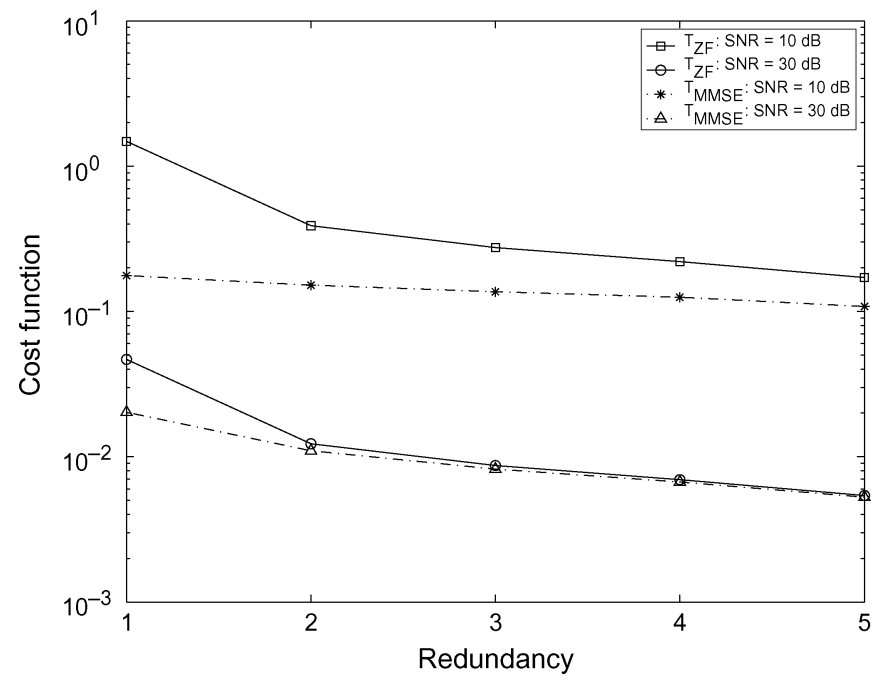

Fig. 1. $T_{\mathrm{ZF}}$ and $T_{\mathrm{MMSE}}$ for different values of redundancy and SNR for $L=$ 4.

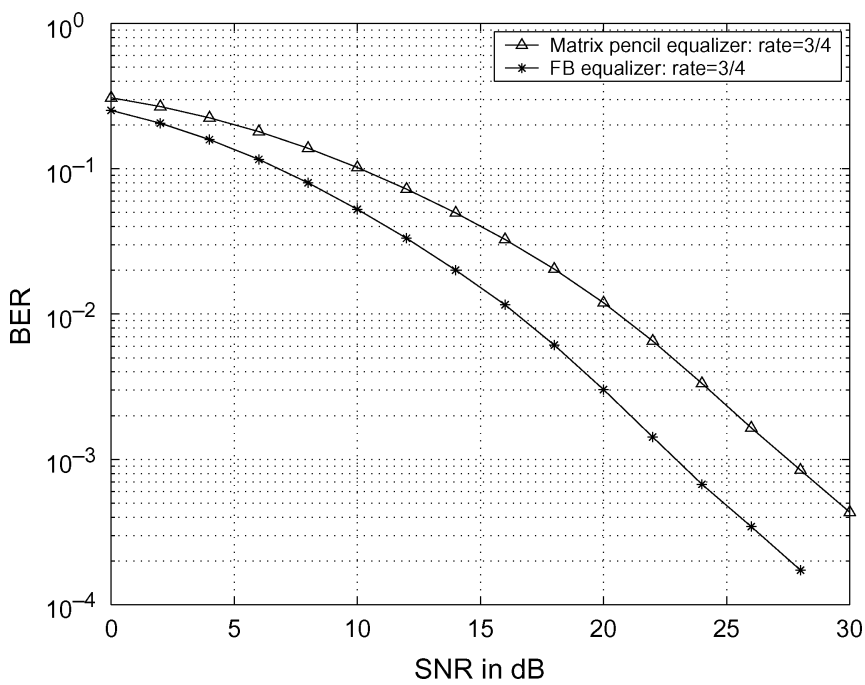

Fig. 2. Comparative performance of FB and matrix pencil-based equalizers.

Fig. 2 shows the comparative performance of FB equalizer and the matrix pencil-based equalizer of [9] for a quarternaryphase-shift-keying (QPSK) input constellation. The redundancy introduced in both cases was one symbol per channel use i.e., $G(z)=\left[\begin{array}{c}I_{3 \times 3} \\ 0_{1 \times 3}\end{array}\right]$. Both the equalizers were ZF equalizers. It can be seen from the figure that the FB equalizer provides a better performance than the matrix pencil-based equalizer.

Fig. 3 shows the comparative performance of the ZF equalizer for different block-lengths $L$ and for different data rates. When $L=4$, and the redundancy introduced is 1 (with $G(z)=$

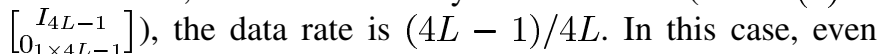
though FIR equalization is achieved, the performance is not satisfactory. However, if the rate is reduced further by increasing the redundancy, as shown in the figure for $L=4$, we see that the performance improves substantially, as could be inferred from Fig. 1, where $T_{\mathrm{ZF}}$ falls sharply when redundancy is increased from 1 to 2 . 


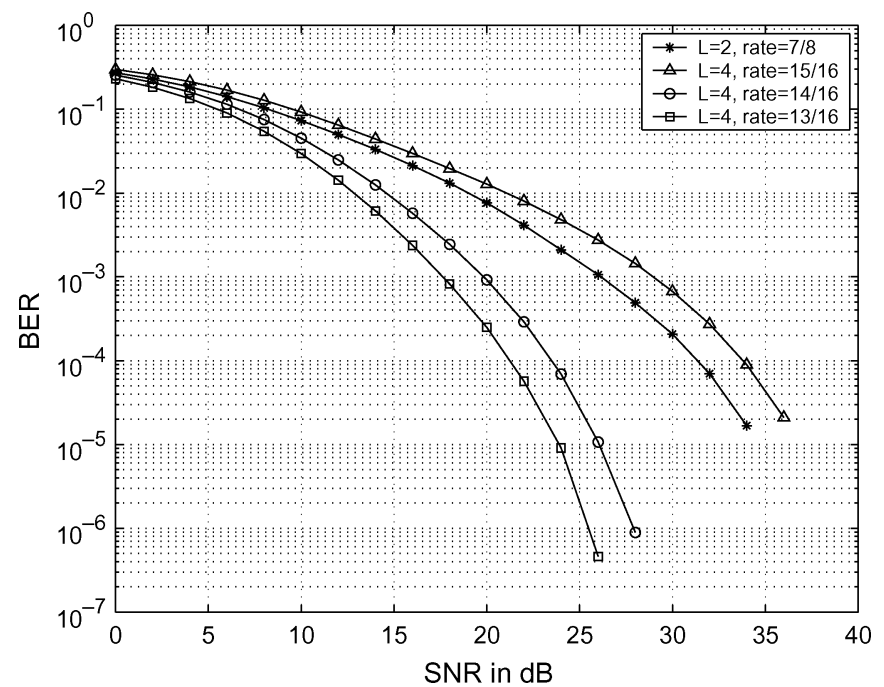

Fig. 3. Performance of MIMO-MCM for different block-lengths and data rates.

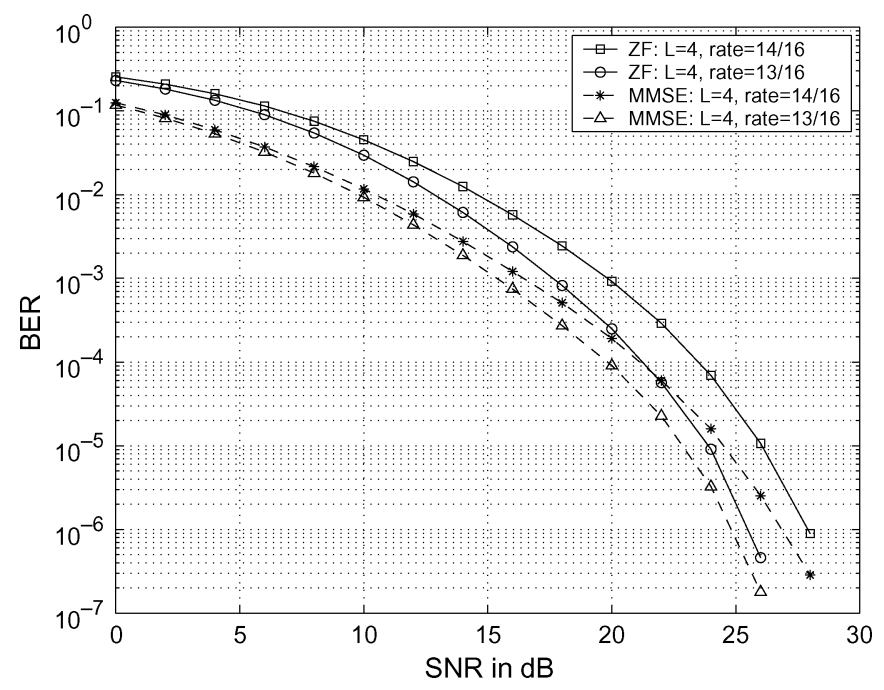

Fig. 4. Comparative performance of ZF and MMSE equalizers for $L=4$.

Fig. 4 illustrates the comparative performance of the ZF and the MMSE equalizers for $L=4$. It can be seen that the MMSE equalizer performs better than the ZF equalizer as expected, and the gap narrows down at high SNR.

Fig. 5 shows the comparative performance of FB precoding with two designs from the ST precoding scheme of [2]. The ST precoder designed according to Lemma 3 of [2] contains as its columns the eigenvectors of $\mathcal{H}^{H} \mathcal{H}$, where $\mathcal{H}$ is the block-Sylvester channel matrix, whereas the design according to Lemma 1 involves power loading across the eigenmodes in addition. The block length was chosen to be 32 . The data rate was $123 / 164=3 / 4$. For the FB precoding scheme, $L=5$, and the rates were $15 / 20=3 / 4,18 / 20=9 / 10$. From Fig. 5, we observe the following.

- For the same rate (3/4), FB precoding performs much better than the ST precoding without power loading (STP-NPL) and on par with ST precoding with power loading (STP-PL). FB precoding with rate $=9 / 10$

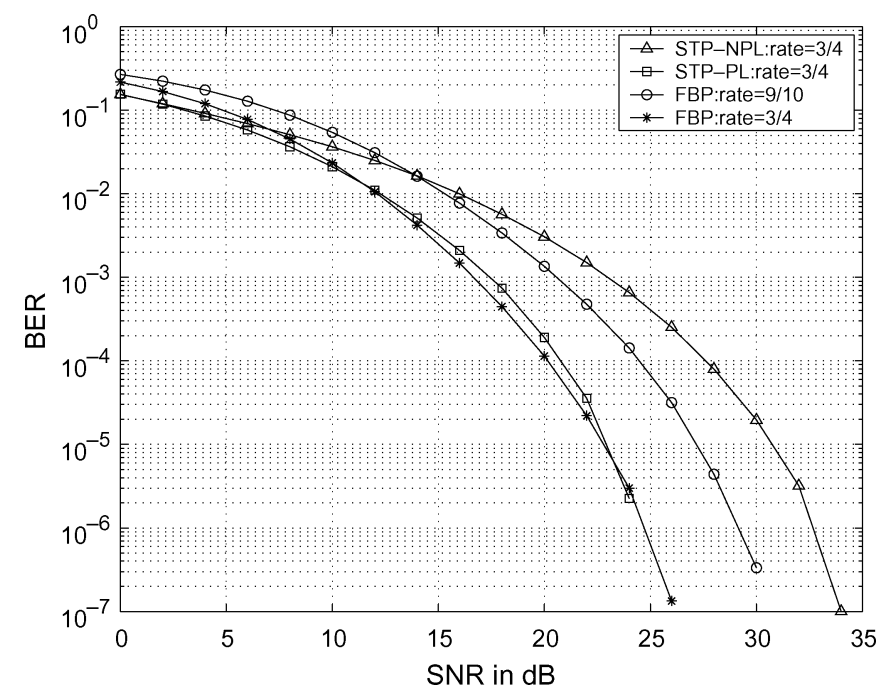

Fig. 5. Comparative performance of FB precoding and ST precoding with ZF equalization. Here, STP-PL $=$ ST precoding with power loading and STP-NPL $=$ ST precoding without power loading.

outperforms STP-NPL with rate $=3 / 4$ at high SNR. Also, no channel knowledge at transmitter was used in FB precoding, whereas ST precoding uses channel knowledge at transmitter.

- The ST precoding scheme has much higher complexity than the FB precoding scheme because of its higher block length. However, it has the advantage of possessing a better structure for a rate-performance tradeoff.

\section{CONCLUSION}

In this paper, we have provided an FB framework for FIR equalization of MIMO frequency-selective channels. We have derived the expression for minimum redundancy required to enable FIR invertibility of arbitrary channels. We have extended the analysis to the case of MIMO multicarrier modulation by investigating the properties of the Smith form of block pseudocirculant matrices. We have also obtained solutions for the optimal ZF and MMSE equalizers. It is shown that the proposed FB precoding approach outperforms the existing ST precoding techniques while supporting a much higher data rate. We have used the simple zero padding precoder in the simulations. Developing optimal precoder designs and the study of sensitivity of the receiver to channel estimation errors are presently under investigation.

\section{REFERENCES}

[1] A. J. Paulraj, D. A. Gore, R. U. Nabar, and H. Bolcskei, "An overview of MIMO communications-A key to gigabit wireless," Proc. IEEE, vol. 92, no. 2, pp. 198-218, Feb. 2004.

[2] A. Scaglione, P. Stoica, S. Barbarossa, G. B. Giannakis, and H. Sampath, "Optimal designs for space-time linear precoders and decoders," IEEE Trans. Signal Process., vol. 50, no. 5, pp. 1051-1063, May 2002.

[3] G. G. Raleigh and J. M. Cioffi, "Spatio-temporal coding for wireless communications," IEEE Trans. Commun., vol. 46, no. 3, pp. 357-366, Mar. 1998.

[4] X.-G. Xia, "New precoding for intersymbol interference cancellation using nonmaximally decimated multirate filterbanks with ideal FIR equalizers," IEEE Trans. Signal Process., vol. 45, no. 10, pp. 2431-2441, Oct. 1997. 
[5] P. P. Vaidyanathan and B. Vrcelj, "Transmultiplexers as precoders in modern digital communication: A tutorial review," in Proc. IEEE Int. Symp. Circuits Systems, vol. 5, May 2004, pp. V-405-V-412.

[6] Y.-P. Lin and S.-M. Phoong, "Minimum redundancy for ISI free FIR filterbank transceivers," IEEE Trans. Signal Process., vol. 50, no. 4, pp. 842-853, Apr. 2002.

[7] H. Sampath, H. Bolcskei, and A. J. Paulraj, "Pre-equalization for MIMO wireless channels with delay spread," in Proc. IEEE Vehicular Technology Conf. (VTC) Fall, vol. 3, 2000, pp. 1175-1178.

[8] J. K. Tugnait, "FIR inverses to MIMO rational transfer functions with applications to blind equalization," in Proc. 30th Asilomar Conf. Signals, Systems Computers, vol. 1, 1997, pp. 295-299.

[9] V. Pohl, V. Jungnickel, E. Jorswieck, and C. von Helmolt, "Zero forcing equalizing filter for MIMO channels with intersymbol interference," in Proc. Int. Symp. IEEE Personal Indoor Mobile Radio Communications (PIMRC), vol. 3, 2002, pp. 1037-1041.

[10] A. Hjorungnes, P. S. R. Diniz, and M. L. R. de Campos, "Jointly minimum BER transmitter and receiver FIR MIMO filters for binary signal vectors," IEEE Trans. Signal Process., vol. 52, no. 4, pp. 1021-1036, Apr. 2004.

[11] B. Vrcelj and P. P. Vaidyanathan, "MIMO biorthogonal partners and applications," IEEE Trans. Signal Process., vol. 50, no. 3, pp. 528-542, Mar. 2002.

[12] P. P. Vaidyanathan, Multirate Systems and Filter Banks. Englewood Cliffs, NJ: Prentice-Hall, 1993.

[13] T. Kailath, Linear Systems. Englewood Cliffs, NJ: Prentice-Hall, 1980.

[14] X.-G. Xia, W. Su, and H. Liu, "Filterbank precoders for blind equalization: Polynomial ambiguity resistant precoders (PARP)," IEEE Trans. Circuits Syst. I, Fundam. Theory Appl., vol. 48, pp. 193-209, Feb. 2001.

[15] J. Boutros and E. Viterbo, "Signal space diversity: A power- and bandwidth-efficient diversity technique for the Rayleigh fading channel," IEEE Trans. Inf. Theory, vol. 44, no. 4, pp. 1453-1467, Jul. 1998.

[16] P. P. Vaidyanathan and S. K. Mitra, "Polyphase networks, block digital filtering, LPTV systems, and alias-free QMF banks: A unified approach based on pseudocirculants," IEEE Trans. Acoust., Speech, Signal Process., vol. 36, no. 3, pp. 981-991, Mar. 1998.

[17] Y.-P. Lin and S.-M. Phoong, "Smith form of FIR pseudocirculants," IEEE Signal Process. Lett., vol. 9, no. 8, pp. 256-258, Aug. 2002.

[18] B. Moore and L. Silverman, "A new characterization of feedforward delay-free inverses," IEEE Trans. Inf. Theory, vol. 19, no. 1, pp. 126-129, Jan. 1973.

[19] P. Loubaton, E. Moulines, and P. Regalia, "Subspace method for blind identification and deconvolution," in Signal Processing Advances in Wireless Communications. Englewood Cliffs, NJ: Prentice-Hall, 2000.

[20] H. Bolcskei, M. Borgmann, and A. J. Paulraj, "Impact of propagation environment on the performance of space-frequency coded MIMO-OFDM," IEEE J. Sel. Areas Commun., vol. 21, no. 3, pp. 427-439, Apr. 2003.

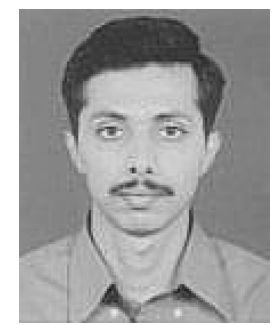

A. Vijaya Krishna received the B.E. degree from the University of Mysore, Karnataka, India, in 1998 and the M.Sc. degree in engineering from the Department of Electrical Engineering, Indian Institute of Science, Bangalore, in 2002. He is currently working toward the Ph.D. degree at the Department of Electrical Communication Engineering, Indian Institute of Science.

From 1998 to 1999, he worked as a Systems Engineer in Wipro Enterprise Solutions. His research interests are in the area of multirate signal processing, signal processing for communications, and MIMO systems.

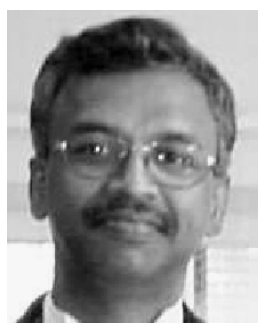

K. V. S. Hari (SM'97) received the B.E. degree from Osmania University, Hyderabad, India, in 1983, the M.Tech. degree from the Indian Institute of Technology, New Delhi, India (IIT Delhi), in 1985, and the Ph.D. degree from the University of California San Diego, La Jolla, in 1990.

Previously, he was a Scientist with the Defence Electronics Research Laboratory, Hyderabad, from December 1985 to July 1987, a Scientist at Osmania University from December 1990 to January 1991, and an Assistant Professor with the Department of Electrical and Computer Engineering, Indian Institute of Science (IISc), Bangalore, India (IISc), from February 1992 to January 1998. Since February 1998, he has been an Associate Professor with the Department of Electrical Communication Engineering, He was a visiting faculty member at Helsinki University of Technology, Espoo, Finland, from May to July 2002; Stanford University, Stanford, CA, from September 1999 to December 2000; and the Royal Institute of Technology, Stockholm, Sweden, from July to September 1995. His research interests are in statistical signal processing. He has worked on space-time signal processing algorithms for direction-of-arrival estimation, acoustic signal separation using microphone arrays, and MIMO wireless communication systems. He has also worked on MIMO wireless channel measurements and modeling and is the coauthor of the IEEE 802.16 (WiMAX) standard on wireless channel models for fixed broadband wireless communication systems. He has been a consultant to various companies around the world and is also a co-founder of a start-up company, ESQUBE Communication Solutions Pvt., Ltd, Bangalore, India.

Dr. Hari is currently a member of the Editorial Board of EURASIP's Journal on Signal Processing. 